\title{
Modeling Transmission Dynamics and Risk Assessment for COVID-19 in Namibia Using Geospatial Technologies
}

\author{
Kedir Mohammed Bushira' ${ }^{1}$. Jacob Otieno Ongala ${ }^{2}$
}

Received: 15 September 2020 / Accepted: 1 February 2021 / Published online: 17 February 2021

(c) Indian National Academy of Engineering 2021

\begin{abstract}
The SARS-CoV-2 infections continue to increase in Namibia and globally. Assessing and mapping the COVID-19 risk zones and modeling the response of COVID-19 using different scenarios are very vital to help decision-makers to estimate the immediate number of resources needed and plan for future interventions of COVID-19 in the area of interest. This study is aimed to identify and map COVID-19 risk zones and to model future COVID-19 response of Namibia using geospatial technologies. Population density, current COVID-19 infections, and spatial interaction index were used as proxy data to identify the different COVID-19 risk zones of Namibia. COVID-19 Hospital Impact Model for Epidemics (CHIME) V1.1.5 tool was used to model future COVID-19 responses with mobility restrictions. Weights were assigned for each thematic layer and thematic layer classes using the Analytical Hierarchy Process (AHP) tool. Suitably ArcGIS overlay analysis was conducted to produce risk zones. Current COVID-19 infection and spatial mobility index were found to be the dominant and sensitive factors for risk zoning in Namibia. Six different COVID-19 risk zones were identified in the study area, namely highest, higher, high, low, lower, and lowest. Modeling result revealed that mobility reduction by $30 \%$ within the country had a notable effect on controlling COVID-19 spread: a flattening of the peak number of cases and delay to the peak number. The research output could help policy-makers to estimate the immediate number of resources needed and plan for future interventions of COVID-19 in Namibia, especially to assess the potential positive effects of mobility restriction.
\end{abstract}

Keywords COVID-19 $\cdot$ Geospatial technology $\cdot$ Spatial interaction index $\cdot$ CHIME modeling $\cdot$ Namibia

\section{Introduction}

The coronavirus disease 2019 (COVID-19) is a contagious disease caused by severe acute respiratory syndrome coronavirus 2 (SARS-CoV-2) (Huang et al. 2020). This virus which is believed to have been originally circulating in wild animals has a similar transmission route with the severe acute respiratory syndrome (SARS) virus (Zhao et al. 2020). It is a respiratory illness with clinical symptoms such as cold,

Kedir Mohammed Bushira

kbushira@nust.na

Jacob Otieno Ongala

jongala@nust.na

1 Department of Civil and Environmental Engineering, Namibia University of Science and Technology (NUST), Windhoek, Namibia

2 Department of Mathematics and Statistics, Namibia University of Science and Technology (NUST), Windhoek, Namibia throat infection, cough, fever, and difficulty in breathing (Huang et al. 2020). The outbreak of COVID-19 was first reported on 31 December 2019, in Wuhan, China (WHO 2020). The virus spread rapidly throughout China and within 1 month, and several other countries, including Italy the United States, Germany, and United Kingdom reported their first cases (Giovanetti et al. 2020; CDC 2020; Rothe et al. 2020). The rapid outbreak and development of the epidemic is contributed to the disease characterization of its long incubation period, high infectivity, and difficulty in detection (Franch-Pardo et al. 2020). In Africa, the first reported case was in Egypt on 25 February 2020. Since then, the outbreak has spread across the continent (Rasheed et al. 2020). Namibia reported its first case on the 14 March 2020 and by the end of June 2020, the total number of confirmed cases remained below 300 . However, it started to increase exponentially from the beginning of July. By the end of July 2020, the number of confirmed cases had reached 2052 (Worldometer 2020). 
As the curve increases exponentially for the number of confirmed cases, Namibia and any other African counties are in worries of possible overwhelming situation in the health care system. The World Health organization (WHO) (WHO 2020) released guidelines to be used to slow down transmission and manage confirmed cases such as case isolation, contact tracing and quarantine, physical distancing, hygiene measures, and improving the health care system. Due to the rapid and continuous spread of the COVID-19 epidemic, several countries or regions all over the world have been forced to take emergency measures such as closing cities, stopping production, suspending school classes, and restricting population movement, causing great harm to economic development and residents' health (An and Jia, 2020). Namibia enforced lockdown of cities from March 2020 and cascaded further control measures guidelines to its region. However, from May 2020 started to relax some measure stage by stage to save its economy (Republic of Namibia 2020). It has now emerged that the epicenter of COVID-19 in Namibia shifted to Erongo Region with more that 50\% of the current confirmed cases (Worldometer 2020). Understanding future dynamics of the disease is important for public health planning and readiness.

Several multidisciplinary studies on the epidemic spread have been done and achieved fruitful research results, which are of great guiding significance for the prevention and control of the epidemic (Franch-Pardo et al. 2020). A comprehensive review by Franch-Pardo et al. (2020) highlighted the importance of health geography in examining health policy interventions, control, and mapping/tracking through projection of spatial diffusion and temporal trends. To achieve this, geographic information systems (GIS) are currently recognized as a set of strategic and analytic tools for analyzing the spread and management strategy to allocate resources for diseases in both developed and developing countries (Wondim et al. 2017). Geography disciplines offer synthetic approach to the interplay between the biophysical and human variables (Turner 2002), and hence, the spatial and temporal changes of the COVID-19 epidemic spread are therefore a scientific problem to study (Xie et al. 2020).

The COVID-19 pandemic have a spatial dimension that lead to understanding the transmission phenomenon as geographical and potentially mappable, and hence the need to include the ability to cross variables of different kinds to interpret the COVID-19 phenomenon, its spatial analysis and spatiotemporal dimensions, its geographical impact on decision-making and everyday life, and predictive modeling of the evolution of the disease (Franch-Pardo et al. 2020). For these reasons, the use of geospatial and statistical tools has become particularly relevant with the declaration of COVID-19 as a global pandemic. Mapping COVID-19 cases will help to understand more about spatial distribution of the disease in their area as well as its temporal occurrence and making forecast of its future burden. Mapping will also be used to locate the areas where outbreaks originate and effectively target high-risk areas for early prevention control. Despite the existence of various challenges of data sources, many countries or regions have published the epidemic spatial models in real time by making use of available data whilst estimating others based on available information (Xie et al. 2020). Sarfo and Karuppannan (2020) assert that geospatial technique is a tool for best practices in fighting COVID-19. In their study, they employed geospatial technologies in Ghana to model trends and mobility patterns. The results forecasted future spread through to the middle parts and then the northern parts. Another study by Ekumah et al. (2020) used a mixture of multivariate statistical and geospatial analyses to investigate the risk of COVID-19 infection in relation to the association of household family structure is associated with in-house access to basic needs in Sub-Saharan Africa (SAA). They used geo-maps to show how high spatial heterogeneity in terms of in-house access to basic needs in SSA. Since the beginning of the pandemic, there is little work done on geospatial research for COVID19 in Africa despite evidence of its application in most parts of the world. This study therefore uses geospatial technologies to model current and future situations of COVID-19 in Namibia.

\section{Materials and Methods}

\section{Namibia}

This study was carried out in Namibia (Fig. 1). Namibia is part of the Southern African countries and has a land area of $825.419 \mathrm{~km}^{2}$. Namibia is surrounded by Angola and Zambezi to the North, Zimbabwe in the Northeast, South Africa to the south, and Botswana to the east and the Atlantic Ocean in the western direction with a heterogeneous population of about 2.4 million.

There are 14 administrative regions in Namibia, with the capital town being Windhoek. Khomas and Ohangwena are the most populous regions. Transport systems, i.e., Harbors, airports, roads, and railways, including the center of each regions of Namibia are shown in the Fig. 1.

\section{Preparation of Input Database}

\section{COVID-19 Risk Assessments and Mapping}

To generate a risk mapping which might help in COVID19 fight of Namibia, data on COVID-19 confirmed cases, population density, and spatial interaction index were collected to prepare different thematic maps. The population density and Namibian road network data were obtained from 


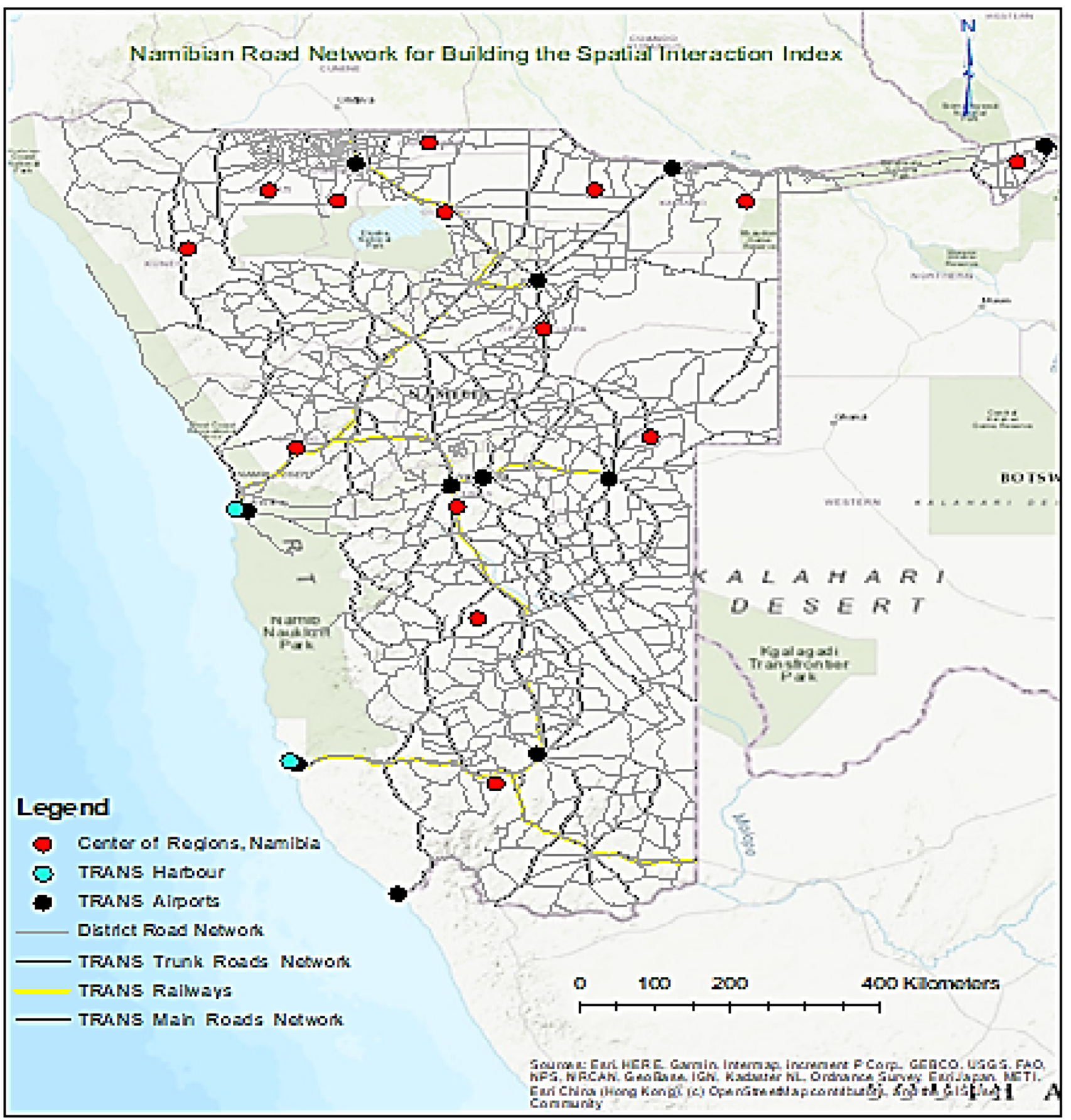

Fig. 1 Contextual map of Namibia

the Namibia Statistics Agency (NSA), National Spatial Data Infrastructure website (http://geofind.nsa.org.na/about). Data on COVID-19 confirmed cases were obtained from Namibia Health Ministry COVID-19 dashboard (https://namib ia.unfpa.org/en/events/health-ministry-launches-covid-19dashboard). On the other hand, the spatial interaction index was generated from a road network data using ARCGIS Pro.
After having prepared all the necessary thematic map layers, each thematic layer based on their relative influence on the spread of COVID-19 was ranked and weighted using AHP pair-wise comparison matrix. Finally, all these prepared thematic layers were integrated using a weighted overlay tool in ArcGIS window to generate the COVID-19 risk assessment and map of the study area. 


\section{COVID-19 Response Modeling}

COVID-19 response modeling was analyzed using ArcGIS Pro COVID-19 modeling toolbox, the COVID-19 Hospital Impact Model for Epidemics (CHIME) V1.1.5 tool. This tool leverages SIR (Susceptible, Infected, Recovered) modeling to assist hospitals, cities, and regions with capacity planning around COVID-19 by providing estimates of daily new admissions and current inpatient hospitalizations (census), ICU admissions, and patients requiring ventilation (COVID-19 response CHIME Model v1.1.5 manual, 2020). The CHIME tool predicts SIR a minimum of 30 days and a maximum of 365 days; however, short-period projections are recommended (COVID-19 response CHIME Model v1.1.5 manual, 2020). In this study, 60 and 90 day projection with and without social distancing was made to analyze the COVID-19 response which might be used by all responsible organizations for a better control of the disease and resource management.

The tool uses parameters that describe the healthcare system or region being analyzed as well as the spread and contact input information for the disease. Spread and contact input information can be specified either as fields in the Input Feature Class or as constant values (COVID-19 response CHIME Model v1.1.5 manual, 2020). All data to run the model were obtained from the Namibia ministry of health website (https://mfl.mhss.gov.na/location-manager/ locations) and tangible information on media briefing by the ministry of health.

\section{More on CHIME Model}

\section{Discrete-Time SIR MODeling of Infections/Recovery}

The model consists of individuals who are susceptible ( $\mathrm{S}$ ), infected (I), or recovered (R).The epidemic proceeds via a growth and decline process. This is the core model of infectious disease spread and has been in use in epidemiology for many years. The dynamics are given by the following three equations (Weisstein 2019):

$S_{\mathrm{t}}+1=S_{\mathrm{t}}-\beta S_{\mathrm{t}} I_{\mathrm{t}}$

$I_{\mathrm{t}}+1=I_{\mathrm{t}}+\beta S_{\mathrm{t}} I_{\mathrm{t}}-\gamma I_{\mathrm{t}}$

$R_{\mathrm{t}}+1=R_{\mathrm{t}}+\gamma I_{\mathrm{t}}$

\section{Parameters}

The model's parameters, $\beta$ and $\gamma$, determine the severity of the epidemic. $\beta$ can be interpreted as the effective contact rate: $\beta=\tau \times c$ which is the transmissibility $\tau$ multiplied by the average number of people exposed $c$. The transmissibility is the basic virulence of the pathogen. The number of people exposed, c, is the parameter that can be changed through social distancing. $\gamma$ is the inverse of the mean recovery time, in days. i.e., if $\gamma=1 / 14$, then the average infection will clear in 14 days.

An important descriptive parameter is the basic reproduction number, or R0. This represents the average number of people who will be infected by any given infected person. When $\mathrm{R} 0$ is greater than 1 , it means that a disease will grow. A higher R0 implies more rapid transmission and a more rapid growth of the epidemic. It is defined as $\mathrm{R} 0=\beta / \gamma$. $\mathrm{R} 0$ is larger when the pathogen is more infectious people are infectious for longer periods of time the number susceptible people is higher. A doubling time of 6 days and a recovery time of 14.0 days imply an R0 of 2.71 (Weisstein 2019).

After the beginning of the outbreak, actions to reduce social contact will lower the parameter $\mathrm{c}$. If this happens at time $\mathrm{t}$, then the effective reproduction rate is $\mathrm{Rt}$, which will be lower than R0 (Weisstein 2019).

\section{The Analytical Hierarchy Process (AHP)}

Multi-criteria decision analysis using the analytical hierarchical process (AHP) is the most common and well-known GIS-based method for delineating risk zones. This method helps to integrate all thematic maps. A total of three different thematic layers were considered for this study. These three thematic layers are supposed to control the factor of COVID19 spread in the area. The association of these influencing factors is weighted according to their reaction for COVID19 spread and expert opinion. A parameter with a high weight illustrates a layer with high impact and a parameter with a low weight illustrates a small impact. The weights of each parameter were assigned according to Saaty's scale (1-9) of relative importance value shown in Table 1 (Satty 1995).

Table 1 Saaty's pair-wise comparison scale

\begin{tabular}{ll}
\hline Verbal judgment & $\begin{array}{l}\text { AHP } \\
\text { numeric } \\
\text { value (scale) }\end{array}$ \\
\hline Extremely important & 9 \\
Very strongly-to-extremely important & 8 \\
Very strongly important & 7 \\
Strongly-to-very strongly important & 6 \\
Strongly important & 5 \\
Moderately-to-strongly important & 4 \\
Moderately important & 3 \\
Equally-to-moderately important & 2 \\
Equally important & 1
\end{tabular}


As per the classification, weights are assigned to the thematic layers based on their importance. Accordingly, all the thematic layers have been compared with each other in a pairwise comparison matrix:

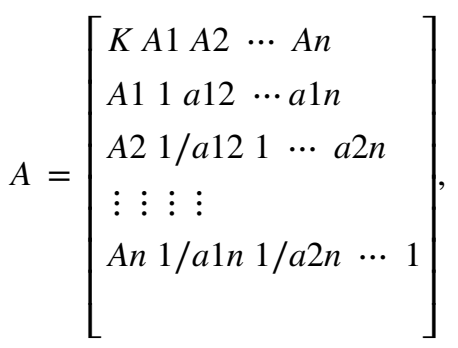

where $A$ is a pair-wise comparison matrix of alternatives $A_{i}$, $i=1,2,3, \ldots n$ with respect to criteria $K$.

The sub-classes of thematic layers were re-classified using natural break classification method in the GIS platform for assigning weight. The sub-classes of each thematic layer rank were allocated on a scale of $0-9$, according to their relative influence on the groundwater development.

For calculating the consistency ratio (CR) (Eq. 4), the following steps are adopted: (1) Principal Eigenvalue $(\Lambda)$ was computed by Eigenvector technique and (2) Consistency Index (CI) was calculated from the equation given below:

$\mathrm{CI}=\frac{\lambda_{\max }-n}{n-1}$,

where $n$ is the number of factors used in the analysis.

Consistency ratio is defined as $\mathrm{CR}=\frac{\mathrm{CI}}{\mathrm{RCl}}$, where $\mathrm{RCI}=$ random consistency index value, whose values were obtained from the Saaty's standard (Table 2).

Saaty has opined that CR of 0.10 or less is acceptable to continue the analysis. If the consistency value is greater than 0.10 , then there is a need to revise the judgment to locate causes of inconsistency and correct it accordingly. If the CR value is 0 , it means that there is a perfect level of consistency in the pair-wise comparison. The threshold value is not exceeding above 0.1 , which means that the judgments matrix is reasonably consistent.

\section{Spatial Interactions index}

Because of their practical prediction performance, trend analysis using spatial interaction index have been preferred for a few decades (Fotheringham and Webber 1980; Champion et al. 1998; Smith et al. 2001). Economy, job opportunity, or industrial structures of a region influence the regional population and its movement (Rogers 2008).

Fundamentally, properties of a region attract population, and its influence is in inverse proportion to the distance. The condition of the regional industry decides the tendency of inter-regional migration, and its amount is determined by the population. It is based on an assumption that people are willing to move to well-structured regions for jobs, market, visit, etc. Those features experience spatial interactions and the terms in the introduced model are modified to reflect them.

The conventional gravity model is composed of the populations of interacting regions, distance, and a constant that decides the strength of the interaction (Smith et al. 2001). The typical form is expressed as:

$I_{\mathrm{ij}}=G_{\mathrm{ij}} \frac{m_{\mathrm{i}} m_{\mathrm{j}}}{d_{\mathrm{ij}}^{2}}$,

where $I_{\mathrm{ij}}$ is the interaction from origin i to destination $\mathrm{j} ; m_{\mathrm{i}}$ and $m_{\mathrm{j}}$ are, respectively, the population functions of regions $\mathrm{i}$ and $\mathrm{j} ; d_{i j}$ is the distance between regions $\mathrm{i}$ and $\mathrm{j}$; and $G_{\mathrm{ij}}$ is a constant determined through statistics of movement from region $\mathrm{i}$ to $\mathrm{j}$.

In this study, the spatial interaction layer was created from road connectivity. To create the spatial interaction layer from the road network, the road network was created using ARCGIS. The spatial interaction index in ARCGIS platform was created using the following procedure.

1. The feature to point tool was used to create point's administrative boundary polygons of Namibia (Fig. 2).

2. Network spatial weights tool setting the Input Feature Class for Namibia administrative points was generated and providing a network data set. The driving distances for each point to every other point were computed. Inverse for the conceptualization of spatial relationships parameter and do not Row Standardize option was selected (Fig. 2).

3. The convert spatial weights matrix to table tool to export the inverse distances to a simple table was used (Fig. 2).

4. Summary statistics to sum the inverse distance weights associated with each administrative boundary was runned (Fig. 2).

5. Join Field to add the summed weights back to the administrative boundaries and alter field to give the joined field an appropriate name such as spatial interactive index was used (Fig. 2).
Table 2 Saaty's ratio index for different values of $N$

\begin{tabular}{lllllllllll}
\hline $\mathrm{N}$ & 1 & 2 & 3 & 4 & 5 & 6 & 7 & 8 & 9 & 10 \\
\hline $\mathrm{RCI}$ & 0 & 0 & 0.58 & 0.9 & 1.12 & 1.24 & 1.32 & 1.41 & 1.45 & 1.49 \\
\hline
\end{tabular}




(1) \begin{tabular}{l}
$(\uparrow$ \\
Parameters Environments \\
Input Features \\
AdminBoundaries \\
Output Feature Class \\
AdministrativePoints \\
$\nabla$ Inside \\
\hline
\end{tabular}

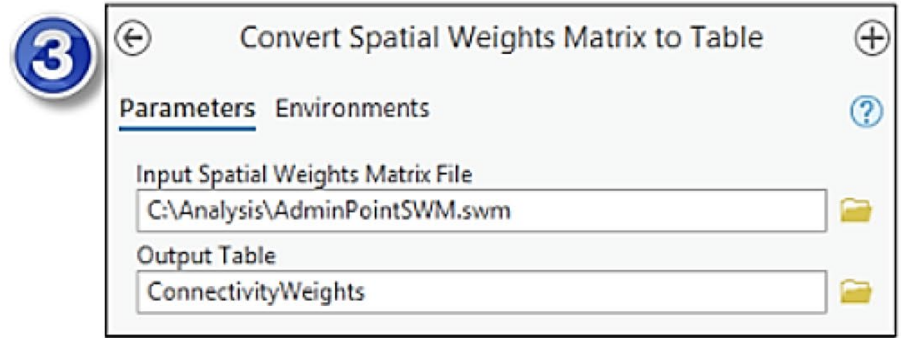

\begin{tabular}{|l|l|}
\hline \multicolumn{1}{|c|}{ Generate Network Spatial Weights } \\
Parameters Environments \\
\hline Input Feature Class \\
\hline AdministrativePoints \\
Unique ID Field \\
\hline ORIG_FID \\
\hline Output Spatial Weights Matrix File \\
\hline C:IAnalysislAdminPointSWM.swm \\
\hline Input Network \\
\hline Routing_ND \\
\hline Travel Mode \\
\hline Driving Distance \\
$\sim$ Network Analysis Options \\
Impedance Cutoff \\
Maximum Number of Neighbors \\
Barriers \\
\hline
\end{tabular}

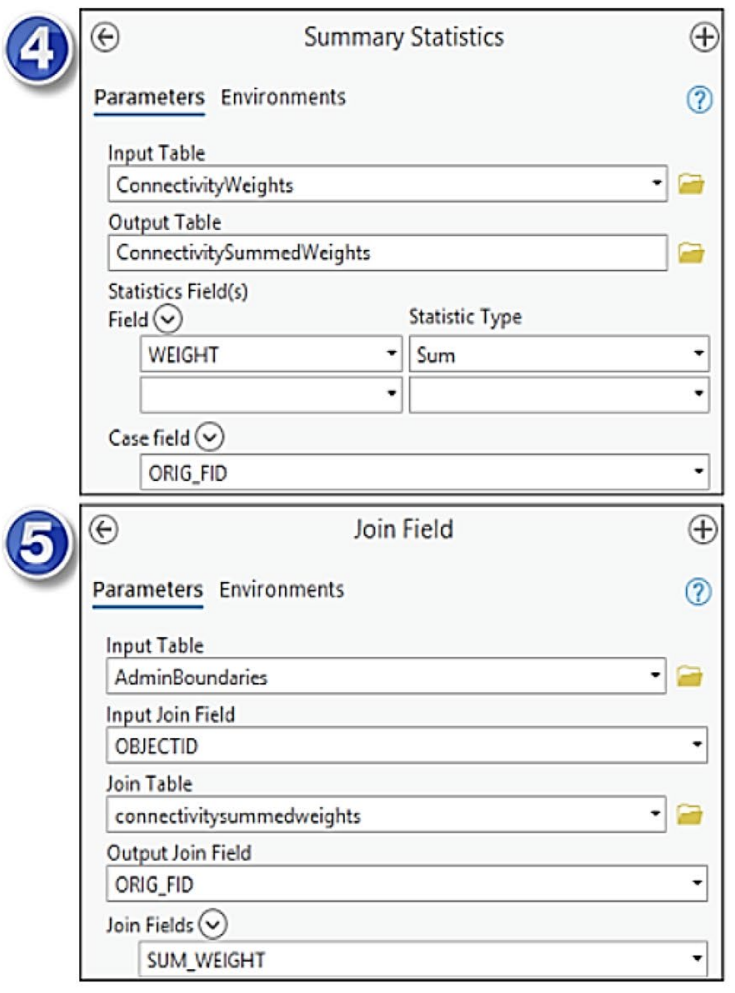

Fig. 2 Steps followed to create the spatial interaction index layer of Namibia

The produced spatial interaction index (Fig. 2) was incorporated in to AHP analysis together with other factors to predict the risk zones. The risk zones in this study can be interpreted only as areas with high transmission of COVID19 and the number of affected population is high in comparisons to the other regions of the country.

\section{Results and Discussion}

In the following sections, the results of the analysis are presented for each of the three factors controlling COVID19 and thereby the risk assessment and mapping. Results on modeling and projections of COVID-19 using CHIME V1.1.5 tool for 60 and 90 days with no social distancing and $30 \%$ social distancing are also presented.

\section{Population Density}

There are 14 administrative regions in Namibia, with the capital town being Windhoek. Khomas and Ohangwena are the most populous regions, while Karas and Omaheke are least densely populated of the 14 regions of Namibia (Table 3).

The population density thematic map was created after forecasting the 2016 population census of Namibia using population growth rate of $1.19 \%$. The population density data are re-classified in to six groups in the ArcGIS Pro platform for overlay analysis (Fig. 3).

\section{Total COVID-19 Cases in Namibia}

Geographically, the spread of the pandemic in Namibia has spatial connotations. From the Table 3, Khomas Region 
Table 3 Population dynamics in Windhoek

\begin{tabular}{llrrll}
\hline No & Region & Population & Total cases & \% Cases & \% Population \\
\hline 1 & Erongo & 85,965 & 2237 & 88.07 & 3.70 \\
2 & Hardap & 182,621 & 9 & 0.35 & 7.85 \\
3 & Karas & 85,862 & 35 & 1.38 & 3.69 \\
4 & Kavango & 148,644 & 2 & 0.08 & 6.39 \\
& east & & & & \\
5 & Kavango & 89,420 & 0 & 0.00 & 3.84 \\
& west & & & & \\
6 & Khomas & 416,279 & 204 & 8.03 & 17.90 \\
7 & Kunene & 97,982 & 1 & 0.04 & 4.21 \\
8 & Ohangwena & 255,817 & 10 & 0.39 & 11.00 \\
9 & Omaheke & 74,719 & 1 & 0.04 & 3.21 \\
10 & Omusati & 250,185 & 2 & 0.08 & 10.76 \\
11 & Oshana & 189,464 & 11 & 0.43 & 8.15 \\
12 & Oshikoto & 195,399 & 2 & 0.08 & 8.40 \\
13 & Otjozond- & 154,527 & 20 & 0.79 & 6.64 \\
& jupa & & & & \\
14 & Zambezi & 98,968 & 6 & 0.24 & 4.26 \\
& Total & $2,325,852$ & 2540 & 100 & 3.70 \\
\hline
\end{tabular}

greatly affected and recorded higher figures next to the hotspot Erongo region. Erongo and Khomas regions have accumulated $88.07 \%$ and $8.03 \%$ of the total COVID-19 cases in Namibia. The third highest hit region makes up just $1.38 \%$ of the confirmed cases. Comparing population distribution and COVID-19 cases (Table 3; Fig. 3), current information's shows that there is no a direct link in infection trends and the regional level of Namibia's population distribution; this might be because of the lockdown measures. Data on confirmed cases were then prepared and re-classified in ArcGIS platform for overlay analysis (Fig. 3).

\section{Mobility Patterns and Spatial Interaction Index}

Population movement triggers transmissions of COVID-19. In this study, Namibian mobility index on the four stages of lockdown was analyzed using Google mobility data (https ://www.google.com/covid19/mobility/). Google calculate these insights based on data from users who have opted-in to Location history for their Google Account, so the data represent a sample of Google users. Google Calculate changes for Groceries \& pharmacy, Retail \& recreation, Transit stations, and Parks categories using the baseline as the median value, for the corresponding day of the week, during the 5-week period Jan 3-Feb 6, 2020. Google mobility index revealed that the average social distancing in the country was 45,21 ,
15 , and $10 \%$ for stage 1 , stage 2 , stage 3 , and stage 4 lockdowns, respectively (Fig. 4). The stage 4 lockdown period is proposed to be extended up to 17 September 2020, but stage 4 mobility change map (Fig. 4) was processed using Google mobility index data till 8 May 2020.

For the COVID-19 risk analysis, the spatial interaction layer of Namibia (Fig. 4) was produced from road connectivity data using ARCGIS Pro. The calculated interaction index then re-classified into six groups for overlay analysis.

\section{GIS Overlay Analysis for COVID-19 Risk Assessment and Mapping}

All the three thematic maps or layers (Fig. 3) were prepared in the re-classified raster format and were given the normalized weight (Fig. 5) in accordance with transmissions of COVID-19. Similarly, each thematic layer's classes were given the normalized rank or weight. Then, overlay analysis was carried out using the weight vectors (Fig. 5) using AHP.

Risk mapping is a dimensionless quantity computed considering the weights for each layer and sub-classes in each thematic layer. After the overlay process has been completed, the COVID-19 risk zone map for Namibia was classified as highest risk, higher risk, high risk, low risk, lower risk, and lowest risk (Fig. 6). Risk zone designated as highest is mostly found in the Erongo Region which stretches from the Central Plateau across to the Central Namibian coast in the west, and with the Ugab River being the northern border, the higher COVID-19 risk zone is distributed along Khomas region which include the capital Windhoek.

High- and low-risk regions are Karas and Otjozonedjupa, respectively. Results also indicate that the lower risk COVID-19 regions are Hardap, Kunene, Oshana, Omusati, Oshikoto, Ohangwana, and Kavango East, and lowest COVID-19 risk areas are Kavango West and Omaheke (Fig. 6).

\section{Future Trend of COVID-19 in Namibia (CHIME V1.15 model)}

The tempo and trend of COVID-19 were modeled using ArcGIS Pro COVID-19 modeling toolbox; the COVID-19 Hospital Impact Model for Epidemics (CHIME) V1.1.5 tool. The modeling was based on mobility dynamics, current COVID-19 cases, population dynamics and the rate of SARS-CoV2 infection, Number of Currently Hospitalized COVID-19 Patients, Social Distancing \% (Reduction in Social Contact), Hospitalization \% (Total Infections), ICU 


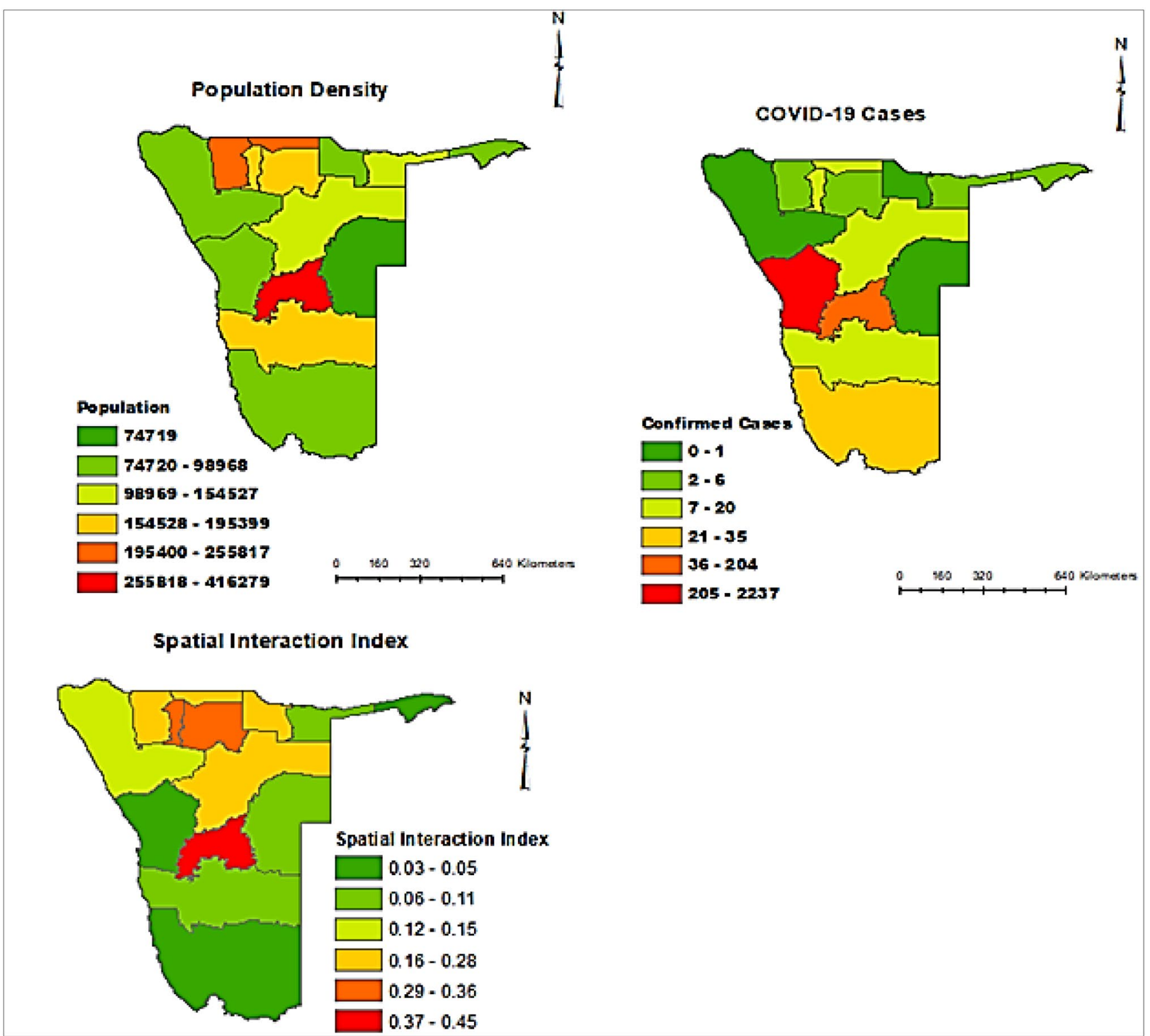

Fig. 3 Population density, confirmed cases, and spatial interaction index for COVID-19 overlay analysis to produce risk mapping

$\%$ (Total Infections), and Ventilated \% (Total Infections), Average Days in ICU, Average Days on Ventilator, Total Bed Capacity, Total Ventilator Capacity, and Total ICU Bed Capacity in Namibia. All data were obtained from different sources and well prepared in ArcGIS pro window in the form of attribute table and imported as spatial data to COVID-19 Hospital Impact Model for Epidemics (CHIME) V1.1.5 tool.

Different scenarios are analyzed and projected for the next 60 and 90 days from the starting date 08 May 2020 with in lock down periods in Namibia. After lockdown ends on 17/ September 2020, the observed data of 18 September 2020 were used for the next 3 -month projections after lockdown ends in Namibia. The metrics explained the maximum difference between projected needs and available resources, including the maximum difference as a total and as a percent, the day, and date in which the highest difference occurred, and the amount of days in which total projected needs exceeded available resources. The 


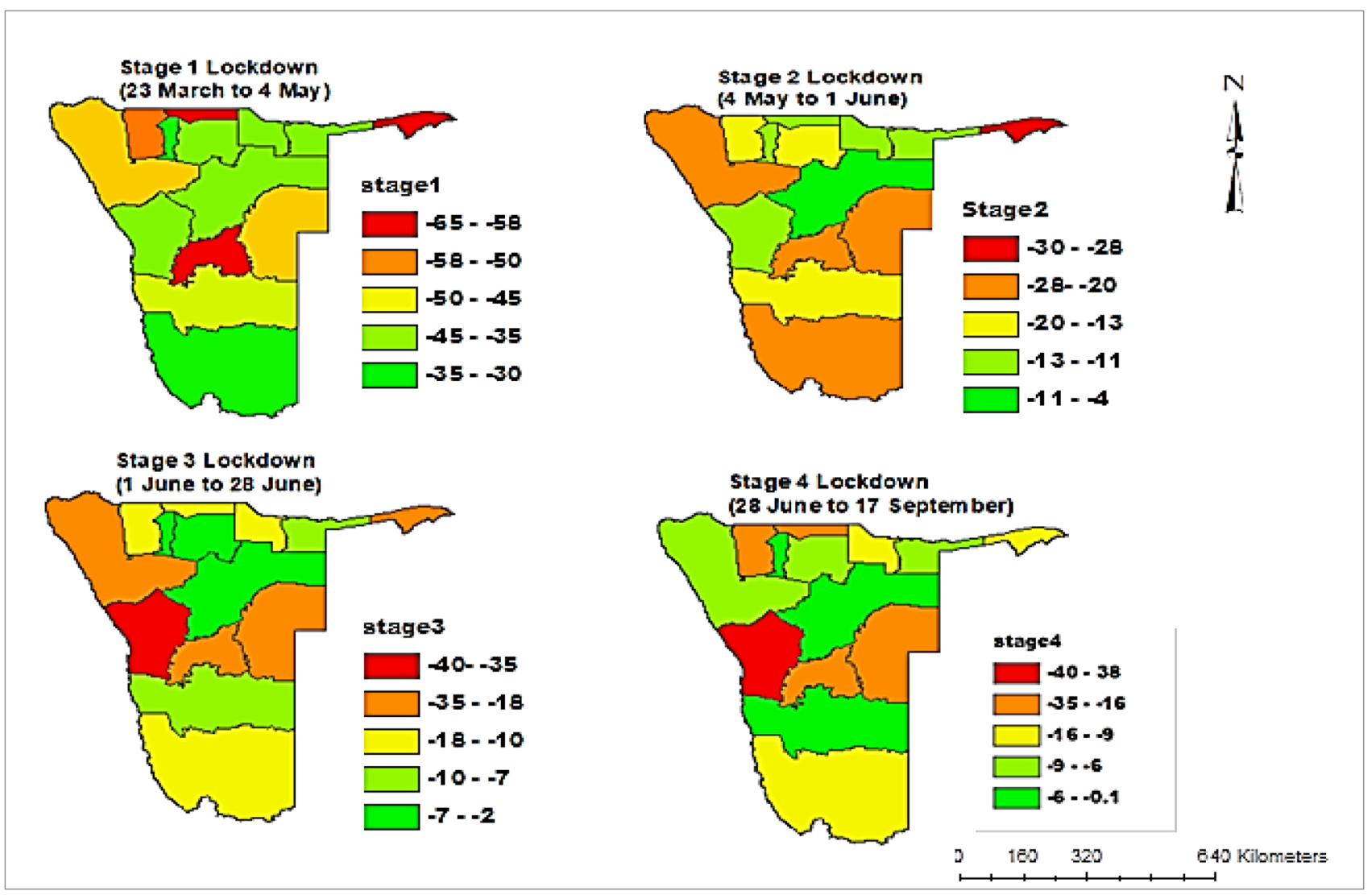

Fig. 4 Regional-level mobility changes during the four stages of lock down in Namibia. Source: Authors Produced based on data derived from Google mobility index 2020

model was processed in four different scenarios to analyze the spread and effect of COVID-19; (1) projection with no social distancing for next 60 and 90 days and (2) projection with $30 \%$ social distancing for next 60 and 90 days. The effectiveness of social distancing interventions to delay or flatten the Epidemic Curve of Coronavirus Disease in Namibia is modeled. Social distancing reduced by $30 \%$.

\section{0-Day Projection: With no Social Distancing and $30 \%$ Social Distancing}

The CHIME model result on transmission of COVID-19 with no social distancing interventions shows a peak curve on new daily admission, daily hospital census, and new hospitalizations for each region of Namibia (Fig. 7). The peak new daily admission will be occurred after 45 days from the starting date $(08 / 05 / 2020)$ of modeling (Fig. 6a). The model result shows about 1524 new peak COVID-19 admissions (Fig. 7a). Out of the new peak admissions, about 400 admissions required ICU and about 200 admissions needed ventilation (Fig. 7a).

The total daily hospital census projections including patients before the modeling period (Fig. 7d) indicate about 10,000 total cases after 50 days of modeling; 3200 ICU admissions and 2100 ventilated admissions.

Khomas region shows high peak about 365 new hospitalizations on 2 September, if no social distance intervention applied (Fig. 7a, c); this is because of the high population density in the region. All other regions have also their peaks on the month of September. The second populous region, Ohangwena, has about 234 newly admission on September 17. The model result reveled that if no social distancing interventions applied, the most populous regions will have the highest COVID-19 cases and high number of COVID-19 cases recorded (Figs. 6c, 7a).

Comparing Figs. 7 and 8, reducing peoples contact rates by $30 \%$ flatten the curve. The new daily admissions and the total daily hospital census projection reduced by $50 \%$. 


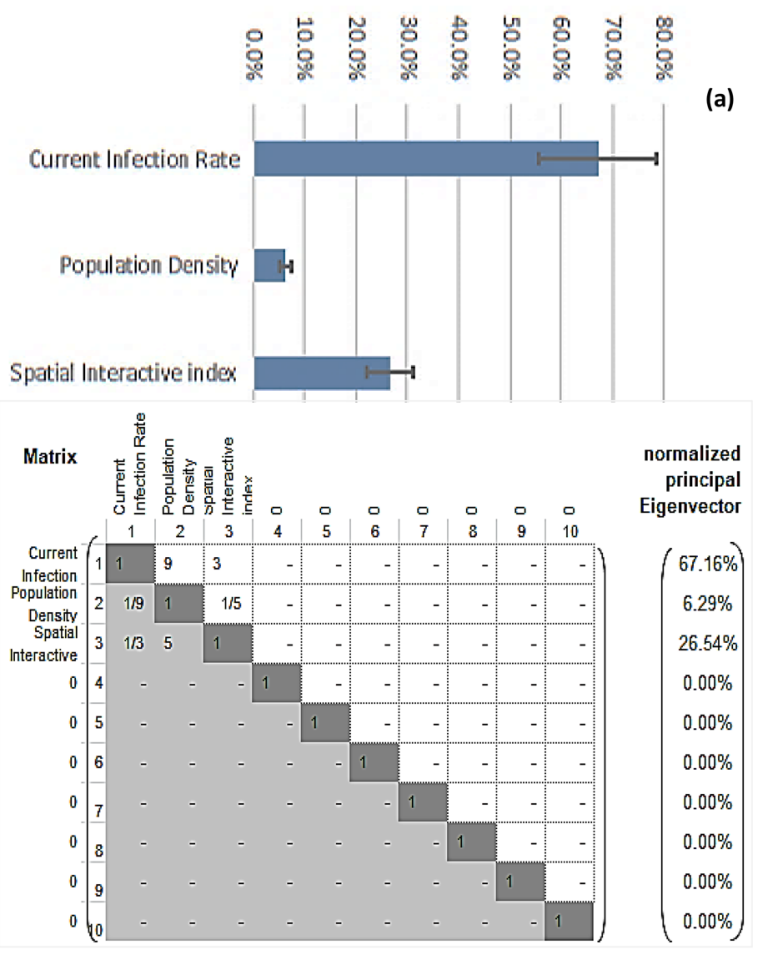

Fig. 5 Percentage influence of factors for overlay analysis and COVID-19 risk mapping

The findings of the model are to be viewed with caution. Hospitalization increases in our model are likely to occur later if measures are lifted or social distance is decreased from $30 \%$ without further action, such as widespread testing, self-isolation of infected individuals, and contact tracing. As with any model, the impact of the interventions may be overestimated by our assumptions. However, quantifying the short-term effects of an intervention is vital to help decisionmakers estimate the immediate number of resources needed and plan for future interventions.

\section{0-Day Projection: With no Social Distancing and $30 \%$ Social Distancing}

Similar to the 60-day model projections above, the 90-day projection also shows high-peak COVID-19 admissions and COVID-19 cases in Namibia with no social distancing interventions scenario (Fig. 9). The model result depicts that newly hospitalization census of each region of Namibia is associated with the number of population (Fig. 9a, c). Khomas region will have the highest peak, about 2500 new admissions during early September. Most of the other

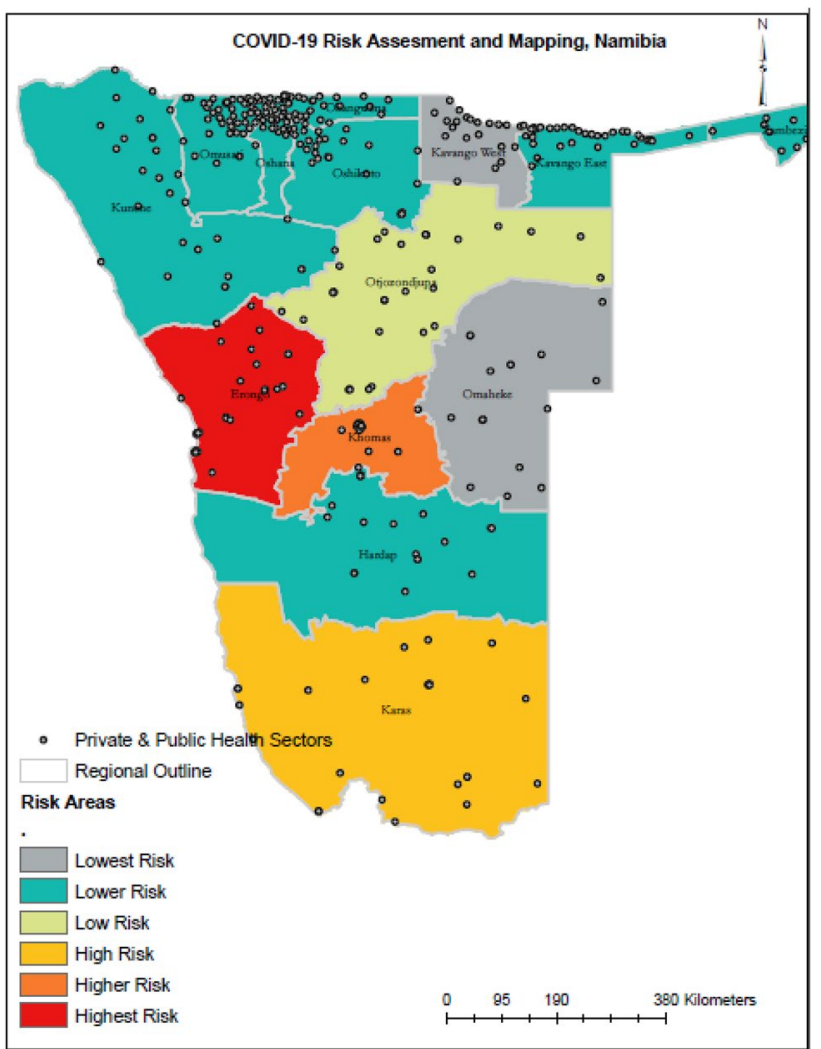

Fig. 6 Regional-based COVID-19 risk zones of Namibia, and IDW interpolation based on the 08 May 2020 confirmed cases. The risk zones in this study can be interpreted only as areas with high transmission of COVID-19 and high number of affected population is in comparisons to the other regions of the country

regions of Namibia will have their peak during late September (Fig. 9c).

The new daily admissions and total daily hospitalized have their peaks after 50 days of projection. Model shows about 1512 new daily hospitalization of which 386 are ICU admissions and about 213 ventilated admissions.

The effect of $30 \%$ social distancing intervention on 90-day COVID-19 response is shown in Fig. 9. Reducing peoples contact rates by $30 \%$ flattens the curve (Fig. 10) in comparison to not applying social distancing measures (Fig. 9).

\section{0-Day Projection Using Data of 18 September 2020: With no Social Distancing}

Since March 2020, 13,134 people have been placed in to mandatory quarantine facilities around the Namibia (Table 4). In Namibia the fourth stage of lockdown ends on 
New Daily Admissions Projections
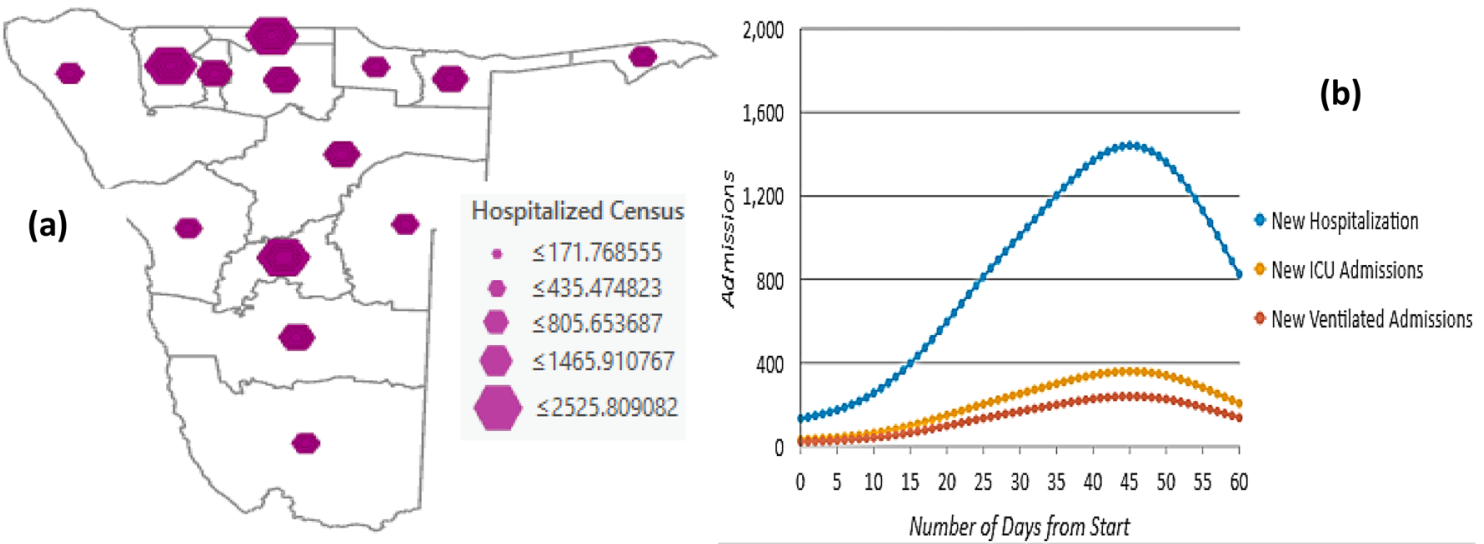

New Hospitalization per Region

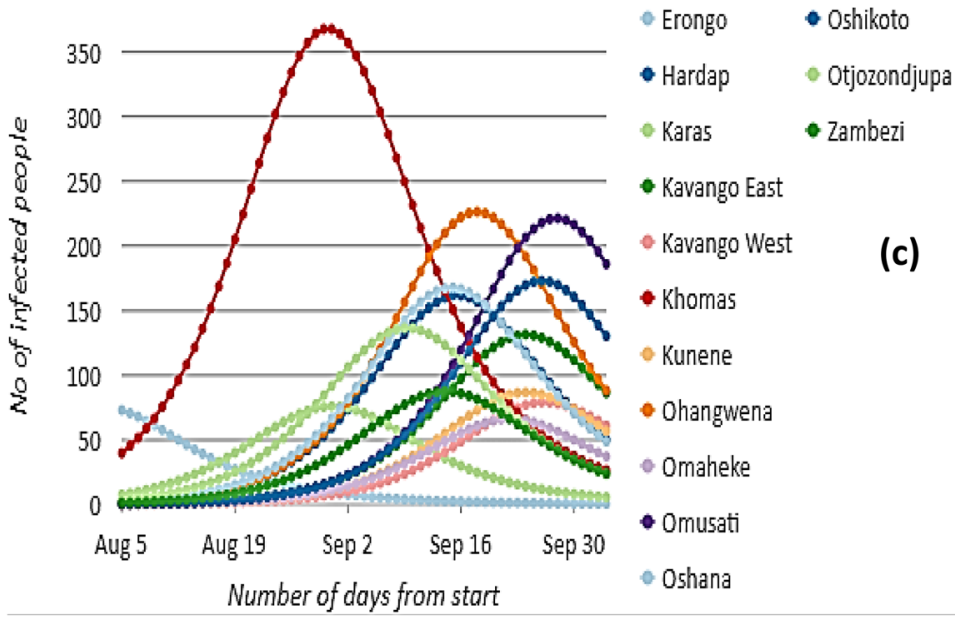

Daily Hospital Census Projections

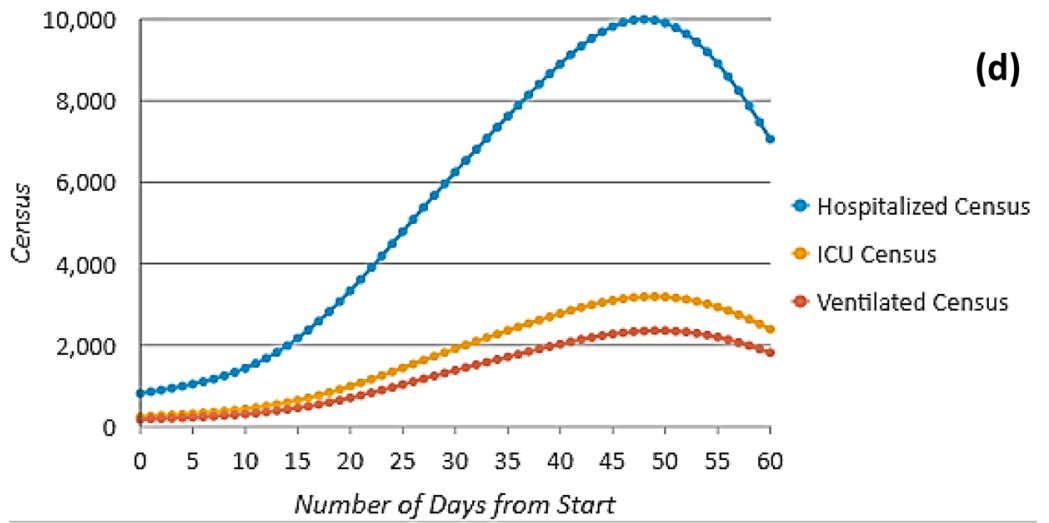

Fig. 7 a Hospitalized census, $\mathbf{b}$ new daily admission projection, $\mathbf{c}$ change in hospitalized census over date per region, and $\mathbf{d}$ daily hospital census projection in Namibia for next 60 days with no social distancing starting from 08 May 2020 


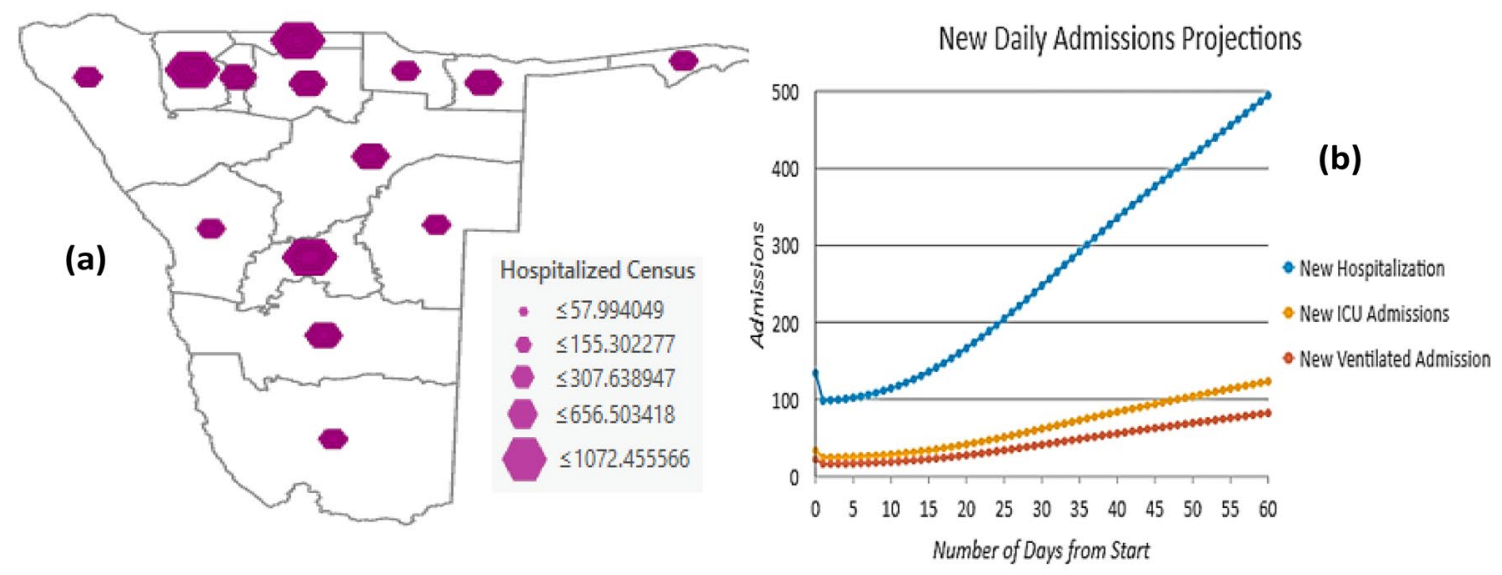

New Hospitalization per Region

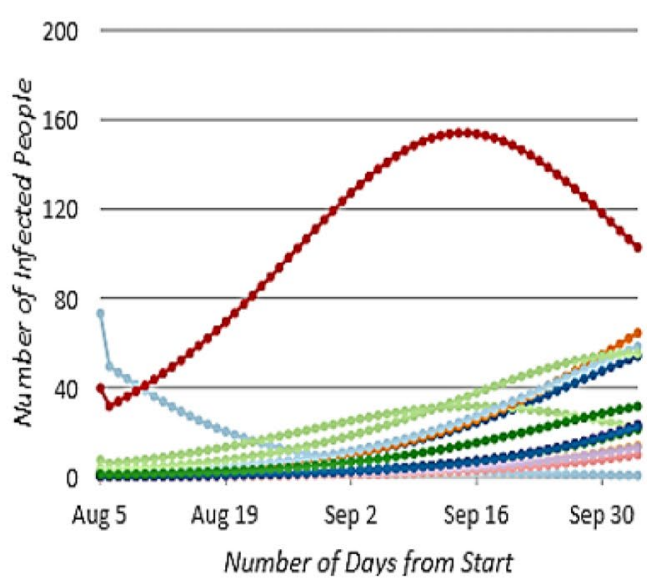

$$
\begin{array}{ll}
\text { - Erongo } & \text { Oshikoto } \\
\text { - Hardap } & \text { Otjozondjupa } \\
\text { - Karas } & \text { Zambezi } \\
\text { - Kavango East } & \\
\text { - Kavango West } & \text { (C) } \\
\text { - Khomas } \\
\text { - Kunene } \\
\text { O Ohangwena } \\
\text { - Omaheke } \\
\text { - Omusati } \\
\text { O Oshana }
\end{array}
$$

\section{Daily Hospital Census Projections}

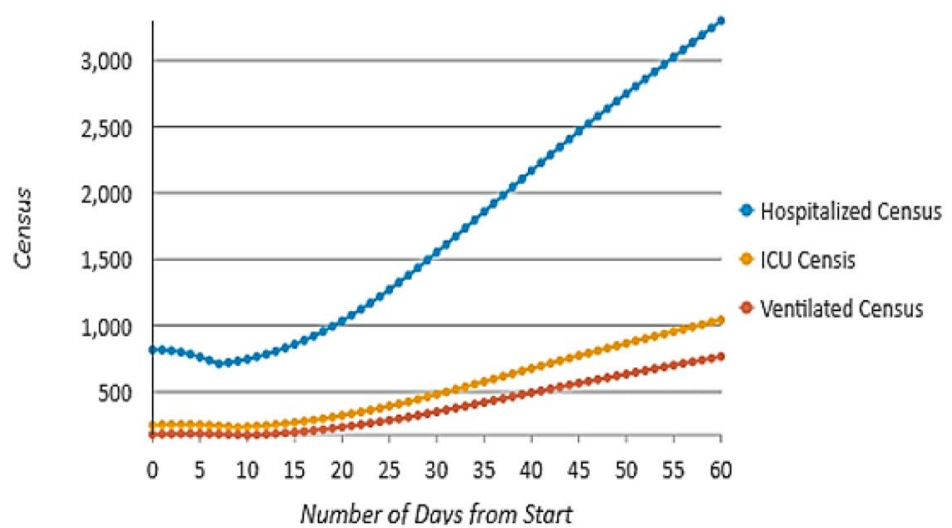

(d)

Fig. 8 a Hospitalized census, $\mathbf{b}$ change in Hospitalized census over date per region, $\mathbf{c}$ new daily admission projection, and d daily hospital census projection in Namibia for next 60 days with no $30 \%$ social distancing 


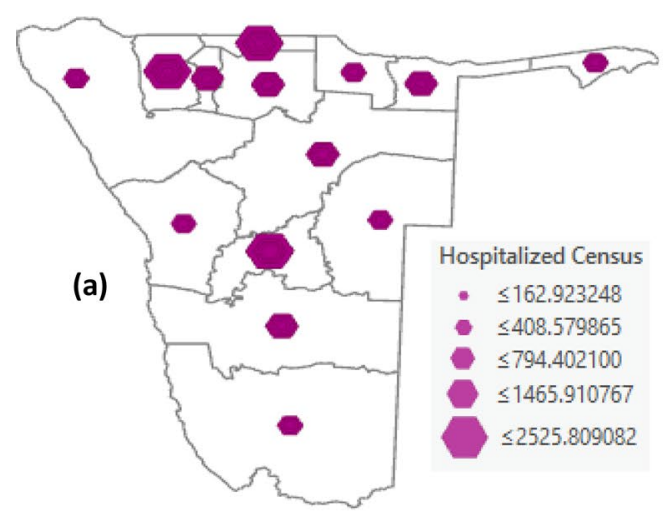

New Daily Admissions Projections

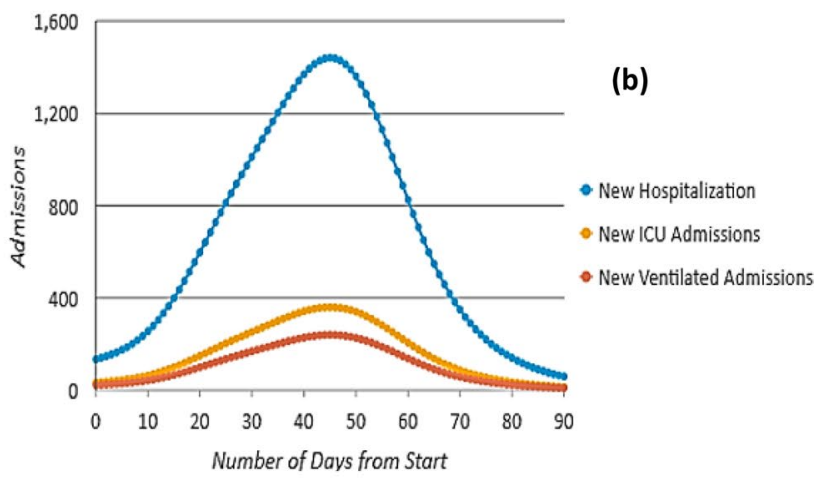

New Hospitalization per Region

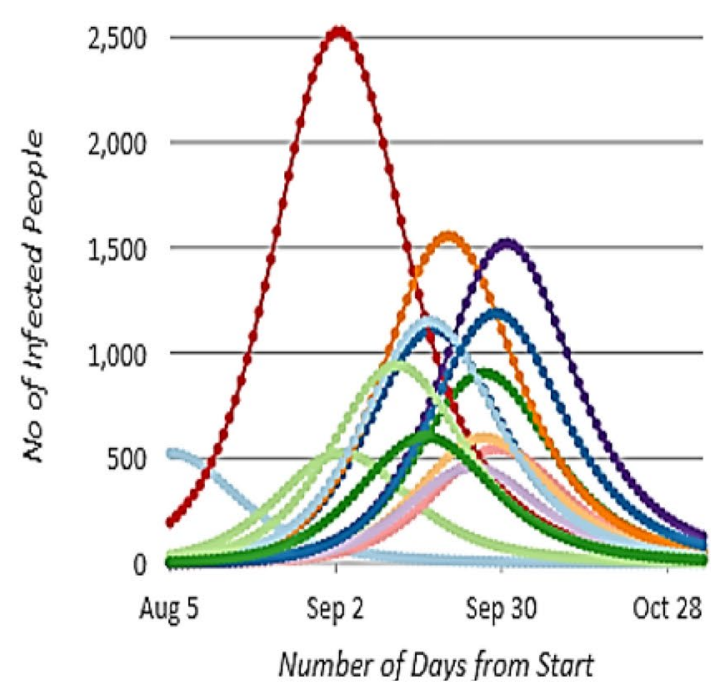

- Erongo Oshikoto
- Hardap Otjozondjupa
- Karas Kambezi
- Kavango East
- Kavango West
- Kunomas
- Ohangwena
- Omaheke
- Omusati
- Oshana

Daily Hospital Cēnsus Projections

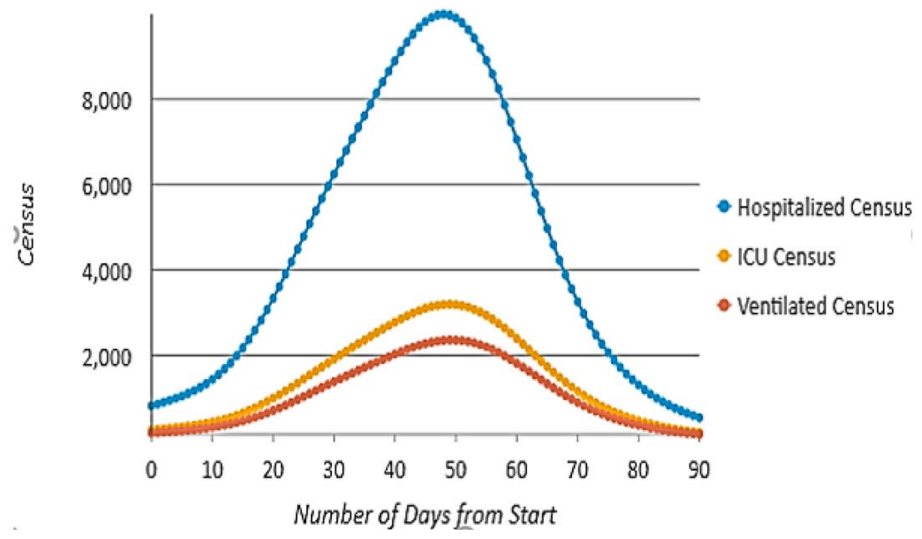

Fig. 9 a Hospitalized census, $\mathbf{b}$ new daily admission projection, $\mathbf{c}$ change in hospitalized census over date per region, and $\mathbf{d}$ daily hospital census projection in Namibia for next 90 days with no social distancing 


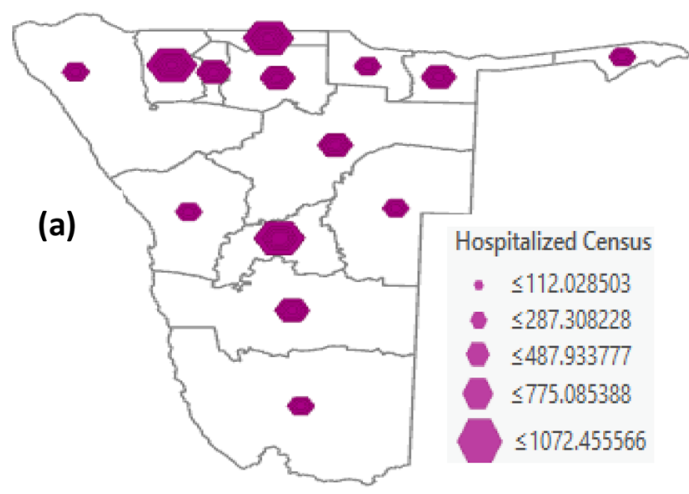

New Daily Admissions Projections

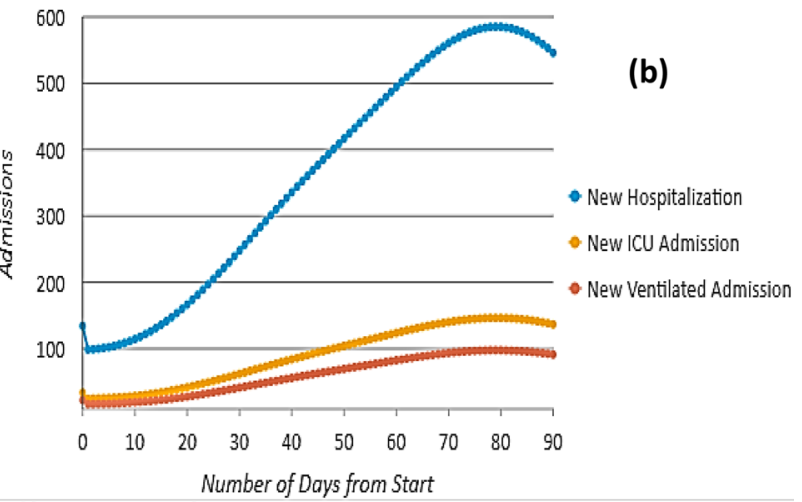

New Hospitalization per region

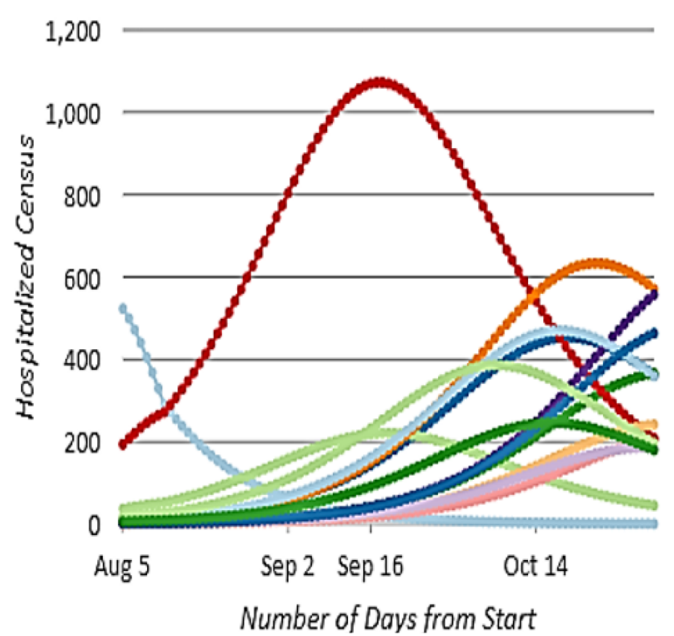

Erongo $\quad$ Oshikoto
- Hardap $\quad$ Otjozondjupa
- Karas $\quad$ Zambezi
- Kavango East
- Kavango West $\quad$ (c)
- Khomas
- Kunene
- Ohangwena
- Omaheke
- Omusati
- Oshana

Daily Hospital Census Projections

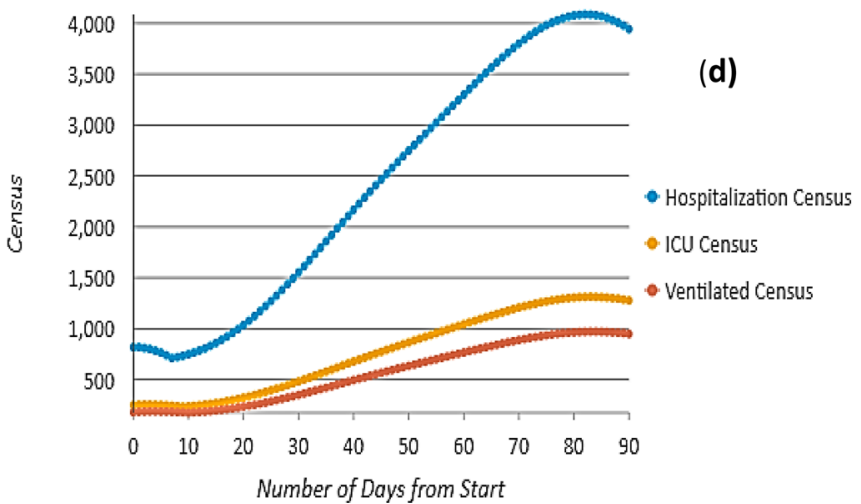

Fig. 10 a Hospitalized census, $\mathbf{b}$ new daily admission projection, $\mathbf{c}$ change in Hospitalized census over date per region, and d daily hospital census projection in Namibia for next 90 days with 30\% social distancing 
Table 4 Observed number of people in mandatory quarantine facilities, 18 September 2020

\begin{tabular}{lcrrr}
\hline Region & $\begin{array}{l}\text { Newly } \\
\text { quarantined } \\
24 \mathrm{~h}\end{array}$ & $\begin{array}{l}\text { Cumulative } \\
\text { Number }\end{array}$ & $\begin{array}{l}\text { Number } \\
\text { discharged }\end{array}$ & $\begin{array}{l}\text { Number in } \\
\text { quarantine }\end{array}$ \\
\hline Erongo & 10 & 3205 & 3126 & 79 \\
Hardap & 10 & 601 & 585 & 16 \\
Karas & 0 & 1680 & 1463 & 217 \\
Kavango & 4 & 439 & 422 & 17 \\
Khomas & 0 & 2259 & 2174 & 85 \\
Kunene & 0 & 439 & 410 & 29 \\
Ohangwena & 5 & 493 & 456 & 37 \\
Omaheke & 29 & 901 & 793 & 108 \\
Omusati & 4 & 294 & 275 & 19 \\
Oshana & 0 & 303 & 294 & 9 \\
Oshikoto & 0 & 884 & 703 & 181 \\
Otjozondjupa & 9 & 838 & 802 & 36 \\
Zambezi & 2 & 708 & 753 & 45 \\
Total & 73 & 13,134 & 12,256 & 878 \\
\hline
\end{tabular}

17 September 2020. 90-Day projection was made using the observed data of 18 September 2020 (Fig. 11).

The 90-day projection reveled Khomas region will have the highest peak, about 4300 new admissions during end of September (Fig. 10b). The projection shows different peak values for each regions due to difference in the no of population and mobility index (Fig. 11b).

New daily hospitalized census projection result shows about 7843 new daily hospitalization of which 2301 are ICU admissions and about 2061 Ventilated admissions (Fig. 11d). More than 1810 new daily admission was also observed (Fig. 11c).

Result findings of this research correlate with an increasing number of publications assessing the impacts of COVID19 interventions. Several researchers have studied how social distancing measures could have influenced the epidemic (Prem et al. 2020; Wu et al. 2020; Kraemer et al. 2020). Others have investigated the effect of similar measures elsewhere and concluded that social distancing interventions alone will not be able to control the pandemic (Flaxman et al. 2020; Tuite et al. 2020). 


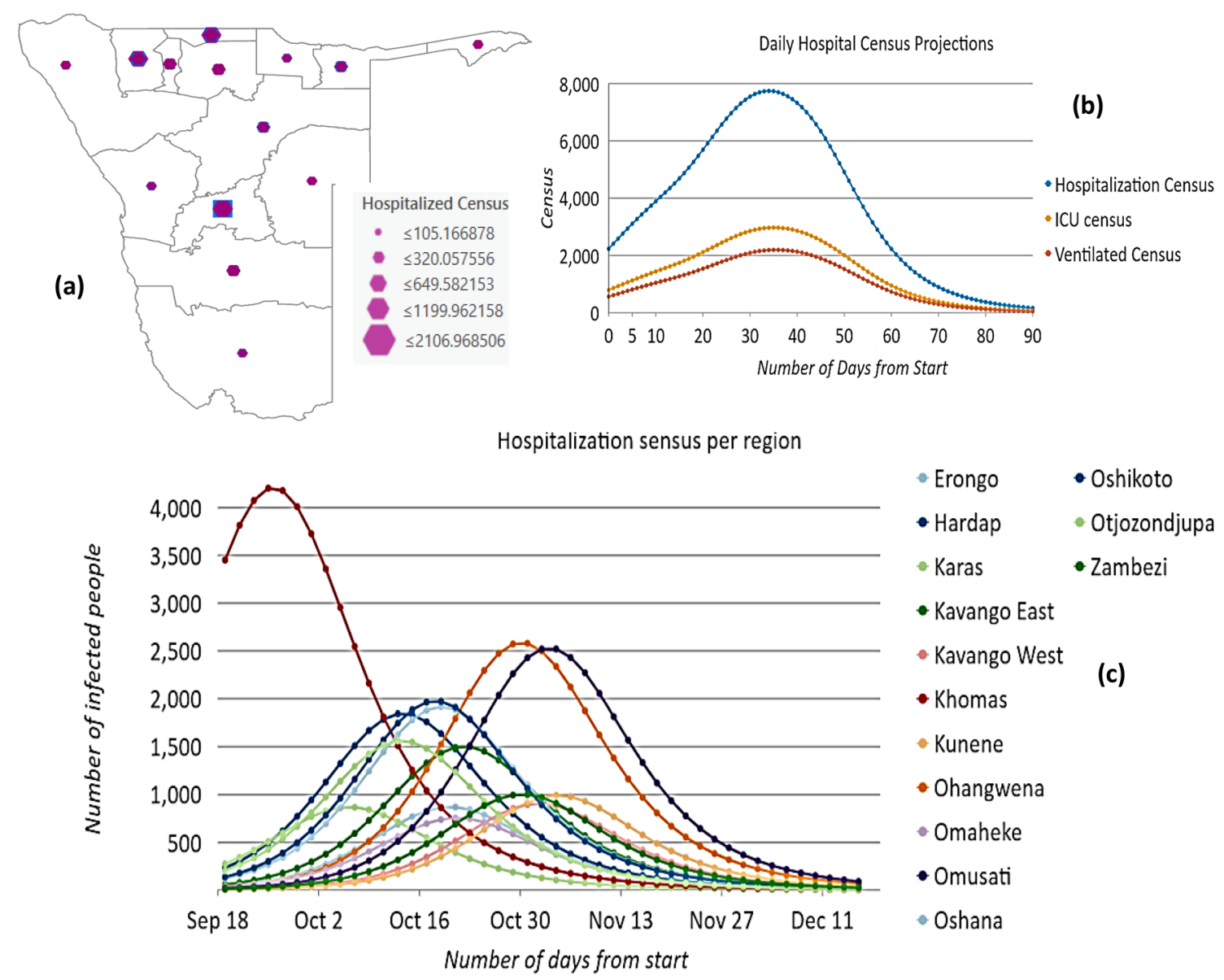

New Daily Admissions Projections

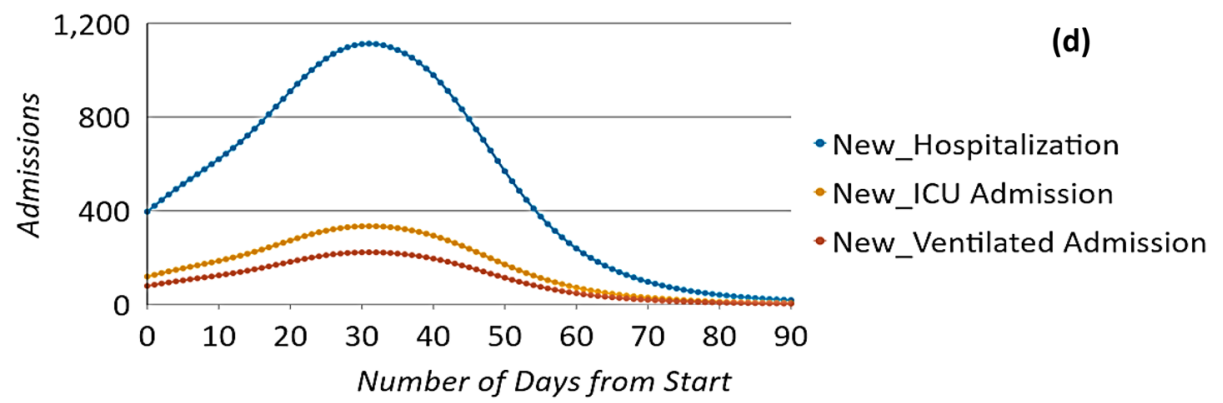

Fig. 11 a Hospitalized census, $\mathbf{b}$ new daily admission projection, $\mathbf{c}$ change in hospitalized census over date per region, and d daily hospital census projection in Namibia for next 90 days from 18 September 2020

\section{Conclusions}

In this study, an attempt was made to develop a spatial model for demarcating the COVID-19 risk zones in Namibia making use of three thematic layers. These were population distribution, current COVID-19 confirmed cases, and spatial interaction index. The COVID-19 risk zones' mapping was produced by integrating these thematic layers in ArcGIS overlay analysis. The identified COVID-19 risk zones in Namibia are highest, higher, high, low, lower, and lowest. Risk zone designated as highest is mostly found in the Erongo Region, the higher COVID-19 risk zone is distributed along Khomas region which include the capital Windhoek, and the high- and low-risk regions are Karas and Otjozonedjupa, respectively. The lower risk COVID-19 regions are Hardap, Kunene, Oshana, Omusati, Oshikoto, Ohangwana, and Kavango East, and lowest COVID-19 risk areas are Kavango West and Omaheke region.

Different scenarios are analyzed and projected for the 60 and 90 days from the starting date 08 May 2020 within 
lockdown periods in Namibia. After lockdown ends on 17 September 2020, the observed data of 18 September 2020 were used for the next 3 -month projections. The CHIME model result on response of COVID-19 was analyzed with and without social distancing intervention for the next 60 and 90 days starting from 08 May 2020. The 60- and 90-day modeling result without social distancing interventions shows a peak curve on new daily admission, daily hospital census, and new hospitalizations for each region of Namibia. The peak new daily admission occurred after 45 days from the starting date of modeling for 60-day projection. The 60-day projection model result shows about 1524 new COVID-19 admissions, about 400 admissions required ICU, and about 200 admissions needed ventilation.

The 60-day model output shows that Khomas region has high peak in September over the other regions; this might be due to the high population density in the area. All other regions have also their peaks on the month of September lately. The second populous region, Ohangwena, has about 234 newly admission on September 17.

The 60 - and 90-day model projection with $30 \%$ social distancing interventions shows flattening of the peak number of cases and delay to the peak number. In Namibia, the fourth stage of lockdown ends on 17 September 2020. After lockdown ends, 90-day projection was made using the observed data of 18 September 2020/.The 90-day projection reveled Khomas region will have the highest peak, about 4300 new admissions during end of September. The projection shows different peak values for each regions due to difference in the number of population and mobility index.

New daily hospitalized census projection result shows about 7843 new daily hospitalization of which 2301 are ICU admissions and about 2061 ventilated admissions. More than 1810 new daily admission was also observed. The research output could help policy-makers to estimate the immediate number of resources needed and plan for future interventions of COVID-19 in Namibia.

\section{Future Scope of the Research}

The worldwide wide spread of COVID-19 peaks the importance of research, stable research infrastructure, and funding for public health emergency, response, and resiliency. Lives are lost, economies falter, and life has radically changed after the pandemic. Ultimate COVID-19 mitigation and crisis resolution are dependent on high-quality research aligned with top priority societal goals that yields trustworthy data and actionable information. While the highest priority goals are treatment and prevention, resource allocation and management require future projections based on the current infection rate.
This study generated a risk zone and projected the new daily admission and daily hospitalized census which might help in COVID-19 fight of Namibia. The projection was mad using two observed data (05 May 2020) and (18 September 2020) for the next 90 days to accommodate CHIME tool projection recommendation. CHIME recommend short-period projections. The model tool and the input parameters can be used for future research and the model can be updated with the recent data at any time possible and future studies can be extended.

The authors recommended that additional data and recent modern technological knowledge which adapts machine learning might increase the accuracy of the research and it is strongly recommended.

\section{Compliance with Ethical Standards}

Conflict of Interest The authors declare that they have no conflict of interests.

\section{References}

An G, Jia F (2020) Analysis of the economic impact of the NCP and countermeasure study. Financ Theor Pract 3:45-51

Centers for Disease Control and Prevention. Coronavirus disease 2019 (COVID-19) in the U.S. [cited 21 Feb 2020]. www.cdc.gov/coron avirus/2019-ncov/cases-in-us.html

Champion T, Fotheringham S, Rees P, Boyle P, Stillwell J (1998) The determinants of migration flows in England: a review of existing data and evidence. Report prepared for the Department of the Environment, Transport and the Regions. The Department of Geography, University of Newcastle upon Tyne, Newcastle upon Tyne, UK, pp 31-128, ISBN 0-902155-39-3

Ekumah B, Armah FA, Yawson DO, Quansah R, Nyieku FE, Owusu SA, Odoi JO, Afitiri AR (2020) Disparate on-site access to water, sanitation, and food storage heighten the risk of COVID-19 spread in Sub-Saharan Africa. Environ Res 189:109936. https://doi. org/10.1016/j.envres.2020.109936

Flaxman S, Mishra S, Gandy A, Unwin HJT, Coupland H, Mellan TA, et al (2020) Imperial College COVID-19 Response Team. Report 13: estimating the number of infections and the impact of non-pharmaceutical interventions on COVID-19 in 11 European countries [Cited 20 Mar 2020]. https://www.imperial.ac.uk/ media/imperial-college/medicine/sph/ide/gida-fellowships/Imper ial-College-COVID19-Europe-estimates-and-NPI-impact-30-032020.pdfExternalLink

Fotheringham AS, Webber MJ (1980) Spatial structure and the parameters of spatial interaction models. Geogr Anal 12:33-46

Franch-Pardo I, Napoletano BM, Rosete-Verges F, Billa L (2020) Spatial analysis and GIS in the study of COVID-19. A review. Sci Total Environ 739:140033. https://doi.org/10.1016/j.scito tenv.2020.140033

Giovanetti M, Benvenuto D, Angeletti S, Ciccozzi M (2020) The frst two cases of 2019-nCoV in Italy: where they come from? J Med Virol. https://doi.org/10.1002/jmv.25699

Huang C, Wang Y, Li X, Ren L, Zhao J, Hu Y, Xu J (2020) Clinical features of patients infected with 2019 novel coronavirus in Wuhan, China. Lancet 395:405-506 
Kraemer MUG, Yang C-H, Gutierrez B, Wu C-H, Klein B, Pigott DM, et al (2020) Open COVID-19 Data Working Group .The effect of human mobility and control measures on the COVID-19 epidemic in China. Science (Epub ahead of print)

Prem K, Liu Y, Russell TW, Kucharski AJ, Eggo RM, Davies N, et al (2020) Centre for the Mathematical Modelling of Infectious Diseases COVID-19 Working Group The effect of control strategies to reduce social mixing on outcomes of the COVID-19 epidemic in Wuhan, China: a modelling study. Lancet Publ Health (Epub ahead of print)

Rasheed Z, Stepansky J, Najjar F (2020). Tracking Africa's coronavirus cases. https://www.aljazeera.com/news/2020/04/tracking-afric a-coronavirus-cases-200401081427251.html

Republic of Namibia (2020) Guidelines for stage 2 under state of emergency-Presidential Statement, Windhoek

Rogers A (2008) Demographic modeling of the geography of migration and population: a multiregional. Perspect Geogr Anal 40:276-296

Rothe C, Schunk M, Sothmann P, Bretzel G, Froeschl G, Wallrauch C et al (2020) Transmission of 2019-nCoV infection from an asymptomatic contact in Germany. N Engl J Med 382:970-971. https://doi.org/10.1056/NEJMc2001468

Sarfo AK, Karuppannan S (2020) Application of geospatial technologies in the COVID-19 fight of Ghana. Trans Indian Natl Acad Eng 0123456789:1-12. https://doi.org/10.1007/s4140 3-020-00145-3

Satty T (1995) Decision making for leaders, 3rd edn. RWS, Pittsburgh

Smith SK, Tayman J, Swanson DA (2001) State and Local Population Projections: Methodology and Analysis. Kluwer, Norwell, pp 97-136 (ISBN 0-306-46493-4)

COVID-19 response CHIME Model v1.1.5 manual, 2020, Version 4-Updated 5/11/2020, The Trustees of the University of Pennsylvania

Tuite AR, Fisman DN, Greer AL (2020) Mathematical modelling of COVID-19 transmission and mitigation strategies in the population of Ontario, Canada. CMAJ (Epub ahead of print)
Turner BL (2002) Contested identities: human-environment geography and disciplinary implications in a restructuring academy. Ann Assoc Am Geogr 92(1):52-74

Weisstein EW (2019) "SIR Model." from MathWorld—a Wolfram web resource. https://mathworld.wolfram.com/SIRModel.html

WHO (2020) Operational considerations for case management of COVID-19 in health facility and community, interim guidance, 19 March 2020. https://www.who.int/publications/i/item/operationa 1-considerations-for-case-management-of-covid-19-in-health-facil ity-and-community)

Wondim YK, Alemayehu EB, Abebe WB (2017) Malaria Hazard and risk mapping using GIS based spatial multicriteria evaluation technique (SMCET) in Tekeze Basin Development Corridor, Amhara Region, Ethiopia. J Environ Earth Sci 7(5):76-87

World Health Organization. Novel coronavirus-China [cited 2020 Jan 12]. https://www.who.int/csr/don/12-january-2020-novel-coron avirus-china

Worldometer (2020) Worldometer COVID-19 data [cited 10 August 2020]. https://www.worldometers.info/coronavirus/country/namib ia/

Wu JT, Leung K, Leung GM (2020) Nowcasting and forecasting the potential domestic and international spread of the 2019-nCoV outbreak originating in Wuhan, China: a modelling study. Lancet 395:689-697

Xie Z, Qin Y, Li Y, Shen W, Zheng Z, Liu S (2020) Spatial and temporal differentiation of COVID-19 epidemic spread in mainland China and its influencing factors. Sci Total Environ 744:140929. https://doi.org/10.1016/j.scitotenv.2020.140929

Zhao X, Li X, Nie C (2020) Backtracking transmission of COVID-19 in China based on big data source, and effect of strict pandemic control policy. Bull Chin Acad Sci 35(3):248-255

Publisher's Note Springer Nature remains neutral with regard to jurisdictional claims in published maps and institutional affiliations. 